\title{
On energy relationships between explosive rupture and extraction processes at quarries
}

\author{
Sergey Zharikov ${ }^{1}$, and Vyacheslav Kutuev ${ }^{1 *}$ \\ ${ }^{1}$ Institute of mining of the Ural branch of the Russian Academy of Sciences, 620075, 58, Mamina- \\ Sibiryaka st., Ekaterinburg, Russia
}

\begin{abstract}
The article deals with the links between mineral extraction processes. The most universal characteristic of the process is its energy intensity. Production process cycles are considered in the relationship between their energy characteristics, taking into account the extraction in the working face over time. This approach has been largely determined by earlier studies in the direction of studying the energy intensity of technological processes. However, the relationship between explosive rupture energy and excavation has not been established, although the link between explosive preparation and the performance of the excavation equipment was stated in the technical literature multiple times. The presented material analyzed previously published materials on establishing relationships between drilling and explosive rupture processes of rock massif. The energy characteristics of excavation works and explosive rupture were considered. Prospective direction of technological links study was proposed. Attention is drawn to the fact that the approach to relationships definition between processes may not always be monotonous, because it is sometimes difficult to express physical meaning in a flat system.
\end{abstract}

\section{Introduction}

As in other spheres of human activity, there are various transients that can also be called processes in the mining industry. For example, in organizing the transport of goods transitions include acceleration and braking of a loaded vehicle. In this case, the process of getting to conventional speed and reducing that speed to a full stop are the most difficult and most dangerous control tasks. This is most clearly expressed in air transportation. In the deposit field developments, transition processes objectively exist not only in the transportation of goods, but in all processes of extraction and processing. This is due to the change in the technological structure as the mining operations deepen and to the full recovery of a particular mineral from the subsoil. The presence of characteristic transition features from some technological techniques to others requires changes in relevant design decisions; they really undergo significant changes during the development period of the whole deposit. Currently, the development of a large deposit is usually designed in orders. Due to this, a lot of tasks are solved in the current development - both in the main processes

\footnotetext{
*Corresponding author: slavik1988@mail.ru
} 
and in the management part, but often the decisions made do not allow for high rates of labor productivity for a long period. Usually it turns out that the introduction of promising technologies, technical devices, control tools is carried out not in a planned manner, but in a spontaneous way, which surely carries a number of problems. The most common is the unpreparedness of staff for new jobs. It happens that the most advanced measurement equipment for the mining machinery data collection is only partially used - only because staff do not know how to interpret the data for achieving a higher quality result of the work. Another problem is often the lack of formalized links between processes. This significantly reduces the coordination of the various enterprise services and negatively affects the management system. The need to study transitions is due to the fact that the innovations introduction in production should not be spontaneous, but planned with appropriate preparatory work [1]. Then special importance in the data sets interpretation is in: establishment of criteria, efficiency indicators of mining and processing processes, as well as appropriate methodological methods for parameters control.

\section{Materials and Methods}

A high organizational degree of mining and processing work is impossible without understanding the links between them. In this regard, the energy characteristics show the processes efficiency and also provide additional data on the development site. If we link blasting and excavation through energy intensity, there may be a formalized connection of the fact known among miners that the performance of the excavator depends on the quality of DBO.

In the research direction sense, the works [2-13] should be noted. Though these works are not directly related, they contain approaches and principles for assessing complex relationships. A number of regularities that allow to approach the estimation characteristics of explosion destruction efficiency and related technological processes are revealed. There are quite a lot of publications regarding the relationship between drilling-and-blasting processes, where the parameters of explosive charges are determined by drilling difficulty and energy intensity. There are publications about energy costs of all mining industry's conversions. However, further and after the explosion, the planned links with the processes seem like they are breaking, although it is noted that in general this connection is present it is just unclear how to define it.

Mined rock loading into the vehicle is a separate issue, which largely depends on experience level and responsibility of the excavator operator. If the handle move at the thrust and lift is smooth, the operation of the mining machine is normal. If the thrust and lift is jerky or the bucket skids in discharge, then the power consumed by the engines changes. With this, the structural elements of the mining machine wear out faster.

The main problem in reducing the energy consumption of processes to a single indicator (such as $\mathrm{J} / \mathrm{m}^{3}$ ) is that the energy consumption of the excavator during mine section discharge is not constant. In determining the average value per $1 \mathrm{~m}^{3}$ for the section as a whole, it is difficult to separate the errors associated with energy costs for the face feeding and transfer of oversized pieces (parts of the mine section). It turns out that the dimensions of excavator's energy consumption depend a lot on various factors, which are currently extremely difficult to take into account. Even with available timekeeping data on one machine with one crew it is difficult to distribute established patterns to other sites, even if the operators' training level is high enough. The properties of the massif and bits and pieces on different quarry sites differ. Approach to solving the issue through measurements can lead to positive results, but only after a long enough data collection on the machines operation at various sites. At the same time, the risk of failure to achieve the expected result in the introduction of measuring equipment is always present. 


\section{Results and Discussion}

Having considered the issue of DBO and excavation relationship, we reached the following conclusion. To at least approximately establish the energy cost of offloading, it is necessary to determine the excavator performance more precisely, which is not always possible. The repair cycle imposes corresponding deviations. At the same time, the connection cannot be established without taking into account the face advance speed, because it is an average indicator of providing the exploded mined rock average piece associated with geometric face parameters. It turns out that in relation to blasting operations it is possible to choose a strict indicator as specific explosive consumption and to determine (taking into account the explosives energy characteristics) energy consumption - as a whole per unit and per cubic meter - and there is no such strict indicator relative to the excavation work. The most accurate is only the cycle time, but it is also difficult to characterize energy costs with it. This is because with face advance the power consumed by the bucket's thrust and lift changes. So, energy costs are determined only in approximation relative to the rated force.

During drilling-and-blasting operations, the energy relationship between processes is reflected in the works [3, 5-7]. According to [7], the specific energy of section drilling-off and the specific energy of the explosive rupture are directly proportional.

An attempt to establish a similar relationship with the excavation works revealed several patterns. In [14], it is shown that when the face advance speed is increased, the total energy intensity of the excavation and explosive rupture decreases; the assumption is made that the former should be considered as criterion in determining relationships, including between further processes. Based on the obtained materials on excavators work modeling, a nomogram was developed (Figure 1) within the boundary conditions of sections volumes to determine the number of excavator cycles depending on the desired face advance speed. This is of high importance in planning mined rock offloading, especially at high intensity of mining operations. 


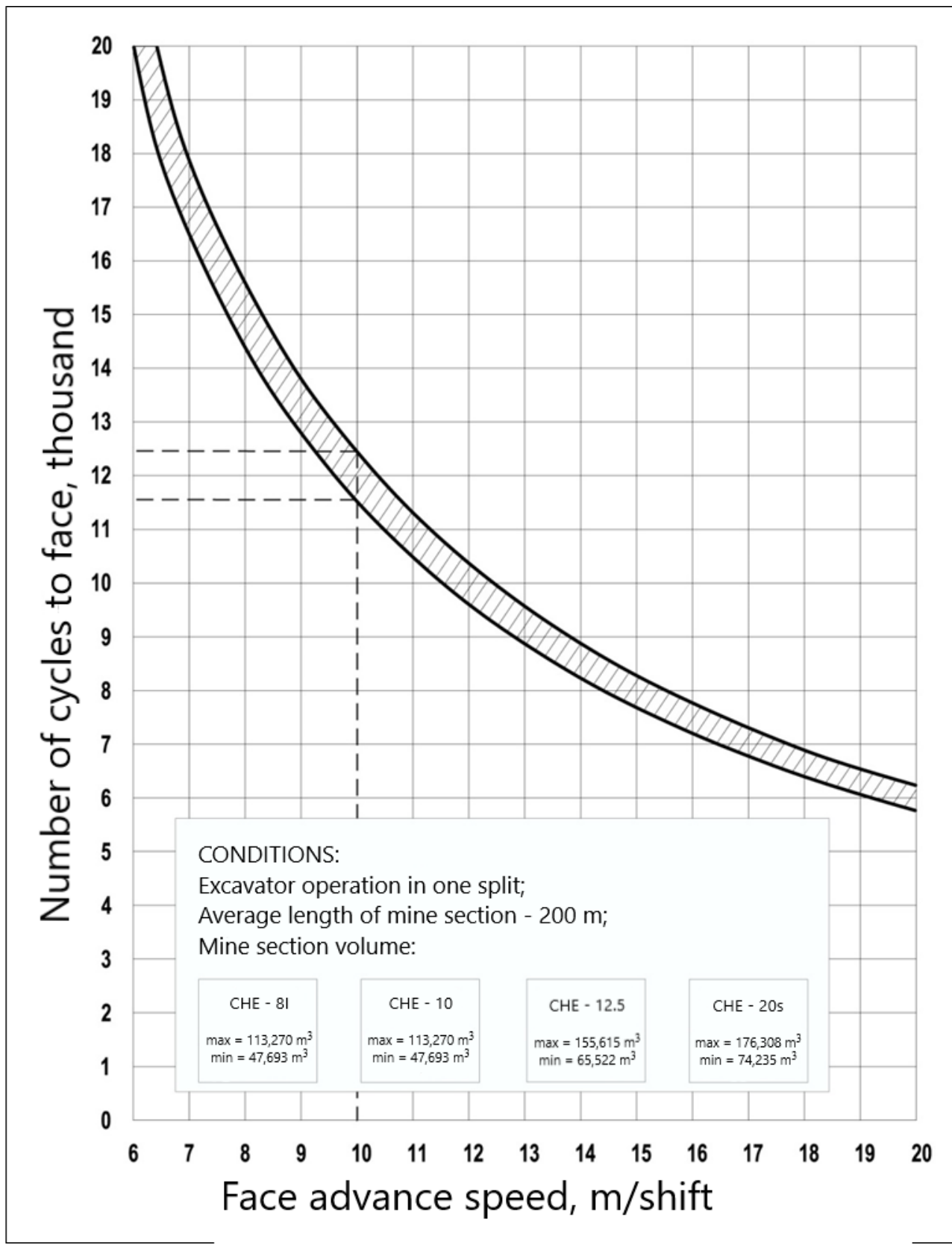

Fig. 1. Nomogram for determining the number of excavator cycles depending on the face advance speed

Currently there is an active movement of mining towards the north, where vast areas have not yet been developed. In the absence of infrastructure and corresponding soil properties of cryolithozone, accelerated mining is the most expedient. This is because it is necessary to reach the production capacity as quickly as possible and further maintain it over time, while preferably not reducing the pace. The approach to deposits development through process intensification probably exsists for objective reasons. However, we must not forget that places with large soils amounts drawoff create large amounts of waste in the 
form of dumps. At the same time, there are usually at least a dozen associated components alongside the main one in the dumps of ore deposits. If we imagine that instead of 1-2 components there will be15-30 extracted from ores, then in many cases the mining intensity is likely to decrease and most importantly — the area for mining waste storage will be reduced. On the other hand, the work intensity itself may not be within the whole quarry, but for example within the quarry site, then when the demand for raw materials is increased, it is easier to increase the production volume. This issue is of some interest to science and production because in any case the work intensity is related to the productivity growth rate.

In the process of further work, it was also found that the full energies of excavation and explosion per section were proportional. For all excavators, the dependencies are similar and have the expression type (1) for CHE-10, only with different numerical coefficients:

$$
E^{e}=0.6062 A^{s}
$$

However, between specific characteristics of the processes it wasn't easy to find the connection right away. The possible causes affecting the achievement of the result were mentioned above. We have suggested that the failure to match specific characteristics of explosion and excavation can be due to the face geometric parameters. To represent the relationship between processes in a flat coordinate system, it was necessary to somehow exclude the section geometry.

When exploding, the energy goes to the massif destruction, which is predominantly unmanageable and is associated with the structural disruption of the latter. When excavated, the energy goes to thrust, lift and turn of the bucket in strict control. Therefore, physically presenting a pattern on a plane is very difficult. The search for a solution has led to the idea that there is such a magnitude as the face advance speed [15] (see expression (2)). It characterizes time, machine design and geometric parameters. There was a need to somehow overcome the latter.

$$
v=\frac{Q}{S},
$$

where $Q-$ the capacity of the excavation complex; $S$ - face area.

During the excavation simulation, the mine section length was almost the same, therefore excluding this parameter and bringing it to a constant was not critical. However, at the same time it allowed to link processes' specific energy costs multiplied by the face area; the latter most strongly expresses the geometric parameters of bits and pieces and also affects the energy intensity [14]. Figure 2 shows the corresponding dependency for the crawler mounted mining shovel with bucket capacity of $10 \mathrm{~m}^{3}$. 


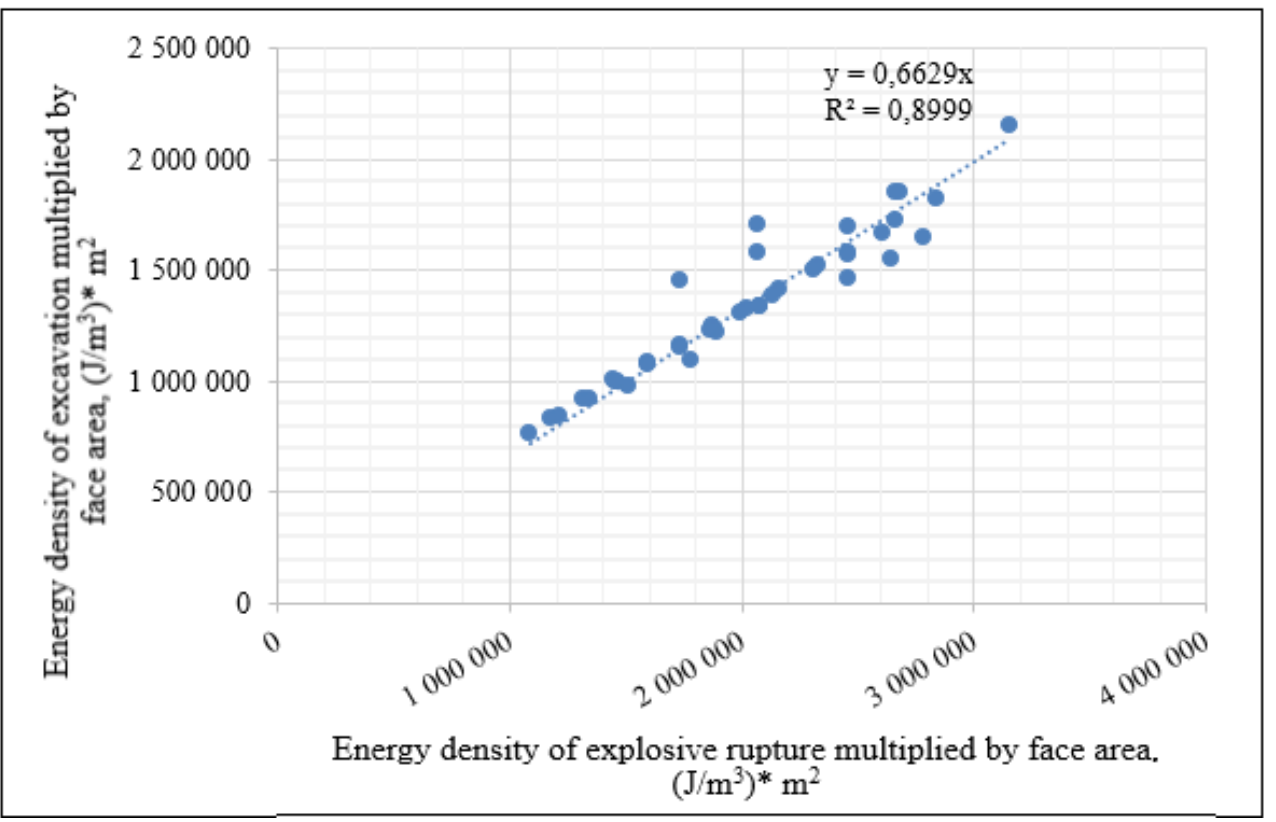

Fig. 2. Dependence of energy consumption on explosion and excavation processes within modelling constraints for CHE-10

For CHE-20s, CHE-12,5, CHE-8I, the dependencies are similar as in Figure 2 with the difference in proportionality coefficient. The drives power was determined when simulating the machines operation in the order set out in [16] with a limitation on the excavator operation in one split. Variants of sections parameters were set within rational proportions with excavating parameters and split width. Processing of data obtained as a result of modeling allowed to express approximate dependence for crawler mounted mining shovel with bucket capacity from $8-20 \mathrm{~m}^{3}$, provided that the face width is less than or equal to the split width (see expression (3)).

$$
E_{s p}^{e} \approx \frac{0.7 A Q}{v S}, \mathrm{~J} / \mathrm{m}^{3}
$$

where $E_{s p}^{e}$ - specific excavation energy, $\mathrm{J} / \mathrm{m}^{3} ; A-$ specific explosion energy, $\mathrm{J} / \mathrm{m}^{3} ; Q$ - excavator capacity $\mathrm{m}^{3} / \mathrm{shift} ; S$ - slack area, $\mathrm{m}^{2} ; v$ - required face advance speed, $\mathrm{m} / \mathrm{shift}$.

\section{Conclusion}

In the studying process of the relationship between blasting and excavation works, the dependence was established between the specific energy of the excavation works with the use of mining shovels with bucket capacity $8-20 \mathrm{~m}^{3}$ and associated values: the specific explosion energy, the excavator performance, the face area and its advance speed. The results obtained are of practical importance for mining planning. Especially in the development and implementation of automated control systems. Depending on the face parameters, the extraction equipment model and the required offloading speed, they allow to approximately set the excavation energy capacity. 


\section{Acknowledgements}

The research was carried out within the framework of the State order, the theme № 0405 2019-0005 (2019 - 2021), as well as with additional attraction of contractual funds.

\section{References}

1. V. L. Yakovlev, Study of transition processes - a new direction in the development of the geo-resources integrated development methodology (UB RAS, Ekaterinburg, 2019)

2. M. A. Sadovskiy, Selected Writings: Geophysics and Physics of Explosion (Science, Moscow, 2004)

3. I. A. Tangayev, Energy of processes and systems of open mining and ore preparation: educational manual (Kyrgyz-Russian Slavic University, Bishkek, Moscow, 2002)

4. V. V. Adushkin, A. A. Spivak, Geomechanics of Large Scale Explosions (Nedra, Moscow, 1993)

5. I. A. Tangayev, Boreability and explosibility of rocks (Nedra, Moscow, 1978)

6. S. N. Zharikov, Bulletin of universities. Mining Journal, 1, 11 (2017)

7. S. N. Zharikov, Relationship of specific energy characteristics of roller-bit drilling and explosive rupture of rock quarry, (IM UB RAS, Ekaterinburg, 2011)

8. Y. Hu, Z. Yang, S. Huang et al., Rock Mech Rock Eng (2020)

9. P. D. Katsabanis, Rock Mech Rock Eng (2020)

10.Physics of Explosion (FIZMATLIT, Moscow, 2002)

11.M. A. Kuk, Industrial Explosives Science (Nedra, Moscow, 1980)

12.G. I. Pokrovsky, Explosion (Nedra, Moscow, 1980)

13.A. Saadatmand Hashemi, P. Katsabanis, Rock Mech Rock Eng 53, 2327 (2020)

14.S. N. Zharikov, Bulletin of universities. Mining Journal, 7, 78 (2017)

15.A. I. Arsentyev, Laws of quarry work zone formation: tutorial (LMI, Leningrad, 1986)

16.K. P. Khmyznikov, Y. V. Lykov, Mining machinery for open mining operations. Mine excavators: tutorial, (Saint Petersburg Mining Institute, Saint-Petersburg, 1999) 